\title{
Prisoners of Abstraction? The Theory and Measure of Genetic Variation, and the Very Concept of "Race"
}

\author{
Jonathan Michael Kaplan • Rasmus Grønfeldt Winther
}

Received: 17 November 2011/Accepted: 27 June 2012/Published online: 20 July 2012

(c) Konrad Lorenz Institute for Evolution and Cognition Research 2012

\begin{abstract}
It is illegitimate to read any ontology about "race" off of biological theory or data. Indeed, the technical meaning of "genetic variation" is fluid, and there is no single theoretical agreed-upon criterion for defining and distinguishing populations given a particular set of genetic variation data. By analyzing three formal senses of "genetic variation," viz., diversity, differentiation, and heterozygosity, we argue that the use of biological theory for making claims about race inevitably amounts to a pernicious reification. Biological theory does not force the concept of "race" upon us; our social discourse, social ontology, and social expectations do. We become prisoners of our abstractions at our own hands, and at our own expense.
\end{abstract}

Keywords Biological ontology · Differentiation · Diversity · Models · Population genetics - Populations . Race $\cdot$ Reification $\cdot$ Variation

The problem for science is to understand the proper domain of explanation of each abstraction rather than become its prisoner (Levins and Lewontin 1980, p. 67).

\footnotetext{
J. M. Kaplan $(\bowtie)$

Philosophy Department, Oregon State University, Corvallis, OR, USA

e-mail: Jonathan.Kaplan@oregonstate.edu

R. G. Winther

Department of Philosophy, University of California, Santa Cruz, CA, USA

e-mail: rgw@ucsc.edu
}

\section{What is at Stake?}

In "But What Are You, Really?" Mills (1988) provides a useful classification of the various metaphysical positions one might hold regarding race (see Fig. 1). At one extreme, Mills suggests that one might be a realist about race, and believe that there are racial "essences," i.e., that members of different races will systematically differ because of features internal and integral to the people making up those races ("essential" to them). Some of these features are morally loaded, including intelligence and personality and character traits, etc.; other characteristics are less morally loaded (or morally neutral), such as various physical characteristics (this is the position that Appiah calls "racialism"). Realism, in Mills' taxonomy, is one of two varieties of objectivism, the view that the existence of race and race categories are independent of our (choice of) beliefs. Constructivism, on Mills' account, is a kind of objectivism, since, while the existence of race and the particular racial categories are determined by our social practices and history, race nonetheless exists (as can be seen by the profound impacts it has on people's lives). For a constructivist, race could have been different, but in fact, it is like this, because this is the way it was made.

At the other extreme from racial realism (racialist beliefs), Mills suggests that one might not just deny the biological reality but the social reality of race as well; this error theory denies the existence of race as both a biological and social category, and hence denies the importance of race. ${ }^{1}$ This view

\footnotetext{
1 This position may seem initially implausible (consider the facts about the relationship between socially identified race and, e.g., health outcomes, noted below), but Mills notes that a certain kind of Marxist, committed to the view that class is explanatory of all important differences in life prospects, might deny the existence of race as a category with any real impact, collapsing the life-prospect issues that follow race into those that follow class (Mills 1988, p. 49).
} 
Fig. 1 The metaphysics of race; redrawn from Mills (1988, p. 45)

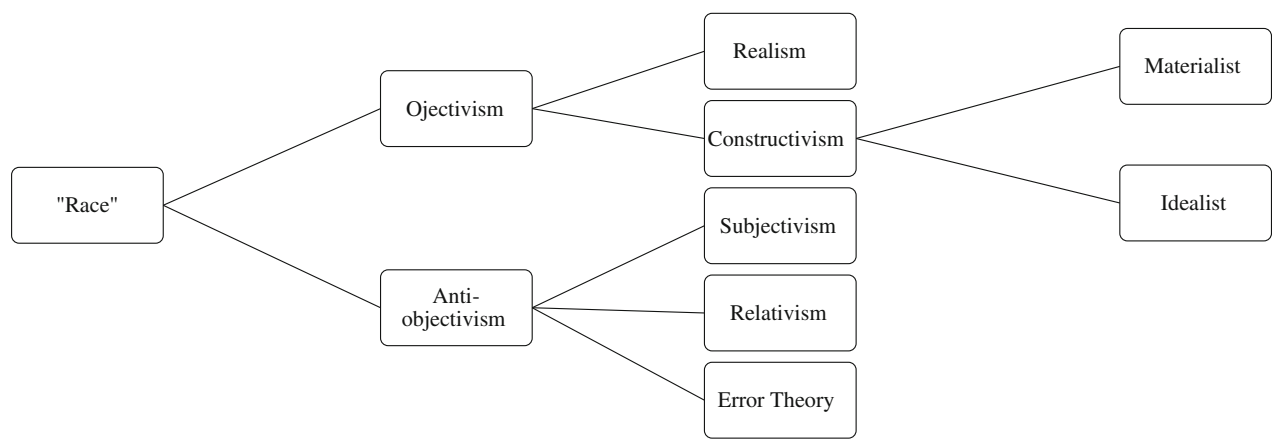

is one of three that deny the "objectivism" of race; race, on these views, is held to be dependent upon our choice of beliefs. Other versions of anti-objectivism explored by Mills include subjectivism (the view that race designations are arbitrary, and can be adopted or discarded freely and voluntarily by individuals) and relativism (the view that races, as entities, depend on the agreement of the populations using the terms).

At first, it might seem the rich sets of theories and metrics associated with population genetics would be decisive, at least with respect to corroborating or falsifying the realist view. After all, if members of a race, on this view, must differ systematically due to features internal and integral to the members themselves, it would certainly seem that the genetics and the biology of population structure - the theory and methods behind cluster analysis and partitioning within- and between-population genetic variation-would be, at the very least, relevant, if not conclusive in adjudicating the existence or not of races. But, we argue, the issue is not so simple.

Indeed, the basic thesis of this paper is that it is illegitimate to read any ontology about "race" — as the term is applied to human groups - off of biological theory or data. In this paper, we focus on epistemic and pragmatic reasons for the illegitimacy, rather than on ethical or political reasons. More specifically, we show that biological theory-in this case, primarily population genetics-cannot ground any claims about the existence or non-existence of race, nor about any sort of biological properties that race may or may not have. In effect, attempting to ground any such substantive claims in biology is a pernicious reification. Our first, overarching thesis is thus that ontological claims about "race" based on biological theory should be avoided, for epistemic and pragmatic reasons: using biological theory to ground race is a pernicious reification. (See also Winther 2011.) In this work, we provide two justifications for this claim.

First, clarity is lacking even within the technical biological literature vis-à-vis the precise, mathematical basis for defining and distinguishing "populations" (or "groups" or "clusters"); biological theory, in the form of mathematical models, underdetermines the entities of biological discourse, and is ill-equipped to deal with the entities of social discourse, such as race. While there is indeed population structure in humans, assessing and explaining exactly how, to what extent, and in what sense this structure exists is not a trivial matter. This should be all the more reason for not applying the categories emerging from particular biological theories to the socio-cultural domain.

In particular, there are a broad variety of formal measures that are used to characterize three distinct aspects of populations, including genetic diversity, genetic differentiation, and genetic heterozygosity. We suggest that, especially in the context of population genetics, insufficient work has been done to clarify the differences and relations among these formal measures, and the sorts of population structures that they each measure and specify. As part of our justification for rejecting the application of biological theory to arguments about race, we argue that definitional and conceptual ambiguity in biological theory should be cleaned up.

Second, as we will discuss in the "Ontological Excesses and Biological Theory" section, the very notion of "race" involves a plethora of social, moral, and political concerns, embedded in particular cultural and historical contexts. There is a complex social discourse to the notion of "race," which varies across different cultural and national contexts, without which we cannot even begin to understand the legitimacy or content of arguments and claims made for the reality (or not) of race. Indeed, genomic data, as well as mathematical population genetic methodologies for interpreting the data, radically underdetermine the particular "racial" classifications used, as well as their legitimacy. Using biological theory for making claims about the existence (or not) of race inevitably amounts to a pernicious reification. Social ontology and discourse are much more explanatory of the use and justification (objective and subjective) of "racial" classifications. Thus, we argue that race is fundamentally a social rather than a biological category.

In short, we intend our analysis to be a constructive contribution to the philosophical and technical literature on human population structure, structure which is indeed 
small but real. We also believe that our warnings are important to rein in any ontological excesses that may flow from the unjustifiable applications of biological theory into socio-cultural questions.

\section{"Race" and Biological Theory}

Claims about the ontological status of race (pro or con), have been grounded in biological theory for centuries. In this section, we briefly sketch a few of these more recent attempts. Our goal here is not to provide a detailed history of the debates surrounding these claims, but merely to give the reader a sense of how particular kinds of claims are said to emerge from particular theoretical commitments in biology.

In a 1962 exchange found in Current Anthropology between the anthropologist Frank B. Livingstone and geneticist Theodosius Dobzhansky, each uses particular claims about biological facts to support their contrasting views on the ontology of human races. Livingstone argues that since most human genetic variation was likely to be clinal in nature and since there were likely multiple different clines along which human populations varied genotypically and phenotypically, it made no sense to think of the human population as being divisible into meaningful races on the basis of genetic differentiation. Livingstone sums up his argument succinctly: "There are no races, there are only clines" (p. 279).

Dobzhansky disagrees. He contends that since the biological race concept meant that populations differed in the "frequencies of one or more genetic variables," and since human populations clearly met this criterion, there were indeed biological human races (1962, p. 280). Dobzhansky admits that if allele frequencies at all loci varied independently from one another, there would be no point in identifying races, since the number of races would equal the number of alleles that varied in frequency. But, he argues, the clines will tend to align and be "steeper where natural, or social, impediments to travel and intermarriage impose obstacles to gene exchange, and more gradual where gene exchange is unobstructed" (p. 280). The number of populations we choose to identify will depend on our interests, but it will not be arbitrary.

This exchange can be framed in the following way: Do we look for sharp boundaries, and, failing to find any, deny the existence of biological populations? Or do we look for clusters-correlated variation-and finding those clusters, affirm the biological reality of the populations so identified? As we will show, there is nothing in the formal theoretical framework of, e.g., population genetics that forces either approach.

We turn now to a more recent "exchange"-Lewontin's (1972) article "The Apportionment of Human Diversity" and Edwards' 2003 response "Human Genetic Diversity: Lewontin's Fallacy." Lewontin's paper was essentially a statistical meta-study that analyzed the structure of genetic heterozygosity in humans, using a Shannon entropy measure (see below). In this analysis, Lewontin found that $85.4 \%$ of genetic variation is found within-populations, $8.3 \%$ is found between those populations that exist within races, and (only) $6.3 \%$ is found across races. From this averaging of the global patterns of heterozygosity structure, Lewontin concluded:

It is clear that our perception of relatively large differences between human races and subgroups, as compared to the variation within these groups, is indeed a biased perception and that, based on randomly chosen genetic differences, human races and populations are remarkably similar to each other....

Human racial classification is of no social value and is positively destructive of social and human relations. Since such racial classification is now seen to be of virtually no genetic or taxonomic significance either, no justification can be offered for its continuance. (1972, p. 397)

Edwards takes issue with this conclusion; he claims that " $[\mathrm{t}]$ here is nothing wrong with Lewontin's statistical analysis of variation, only with the belief that it is relevant to classification" (pp. 800-801). Edwards argues that the data cited by Lewontin cannot support the claim that "the division of Homo sapiens into these groups [i.e., races] is not justified by the genetic data" (2003, p. 798). While population structure is not revealed by the total, averaged variation, it can be identified by the correlations among the individual variations in allele frequencies. Even small differences in, e.g., allele frequencies between populations can be used to reveal population structure, given sufficient loci (2003, p. 801). Edwards interprets Lewontin as denying the existence of population structure in humans, ${ }^{2}$ and provides a statistical critique of that interpretation-a critique he suggests is borne out by the recent successful work in genetic clustering analysis (e.g., Pritchard et al. 2000; Rosenberg et al. 2002, both cited by Edwards 2003; Kalinowski 2011 provides a recent criticism of this work).

Lewontin suggests that "race," as it is used in ordinary social discourse, carries implications about the existence of sizeable inborn differences between members of different races as well as significant similarity between members within races; finding no such sizeable differences between

\footnotetext{
${ }^{2}$ Lewontin (1972) does not explicitly deny the existence of population structure in humans-indeed, we are unaware of any biologist who has done so. But his statistical results and criticism of the "race" concept as socially destructive and as (nearly) biologically useless are often interpreted this way, and it is this interpretation that Edwards seems to have in mind. See Kaplan (2011) for discussion.
} 
races and finding significant variation within races, Lewontin argues that the ordinary meaning of "race" is undermined by biology. A theory about what "race" means is confronted with a theory about how to partition genetic variation, and race, as a category, is found wanting. Edwards rejects this line of reasoning. Taking the question to be about the existence of identifiable population structure-an entity relevant to a different part of biological theory-Edwards argues that what matters is whether population structure can be teased out of the data. Since it can, he argues that the argument that "race" as a category is undermined by biology fails.

Again, demes (local populations whose members preferentially interbreed) are not races, and the existence (or non-existence) of this social, moral, and political category (or set of categories) should not and cannot be read off the existence (or non-existence) of population structure in nature. To make the point as bluntly as possible: there is population structure in Homo sapiens, even if we are also, to a "zeroth" approximation, all genetically identical. ${ }^{3}$ However, that there is a population structure fails to provide epistemic and pragmatic grounds about the reality or justification of those socio-political categories about which there is so much discussion, race. The "Diversity, Differentiation, and Heterozygosity as Distinct Metrics and Meanings of Genetic Variation" and "Ontological Excesses and Biological Theory" sections below are dedicated to showing whence such failure.

\section{Diversity, Differentiation, and Heterozygosity as Distinct Metrics and Meanings of Genetic Variation}

The above explores the uses that have been made of the limited genetic variation in contemporary humans, as well as the history and discourse surrounding such uses. In this section we explore the technical methodologies used to

\footnotetext{
3 Contemporary humans vary, on average, by about $1: 1,000$ nucleotides; this is around an order of magnitude less variation than occurs in many other species (see, e.g., Li and Sadler 1991; Cognato 2007). All the measures of genetic variation discussed below rely on this small amount of variation when applied to humans. This variation is not distributed equally in humans; people whose ancestors are of recent African origin, for example, differ on average by about 1:900 nucleotides; people whose ancestors were of recent European origin differ by only about 1:1,600 nucleotides. Edwards is correct when he notes that "It is not true, as Nature claimed, that "two random individuals from any one group are almost as different as any two random individuals from the entire world" "(2003, p. 801) but not perhaps in the way he intended; interestingly, the "average" person of recent African descent is more likely to share an arbitrary allele with an "average" person of recent European descent (will differ less) than he or she is with another arbitrarily chosen person of African descent (African v. European difference is about 1:1050, compared with 1:900 difference within Africa) (see Yu et al. 2002).
}

measure genetic variation. There are many of them, and their aims and biological interpretations, as well as the relationships among them, are often unclear. ${ }^{4}$ We wish to clarify definitions and concepts of genetic variation, and examine how they relate to one another, by proposing a classification of varieties of measures of genetic variation, which distinguishes genetic differentiation, genetic diversity, and genetic heterozygosity. ${ }^{5} \mathrm{We}$ intend to make explicit hidden methodological and theoretical assumptions and meanings.

Consider first the distinction between diversity and differentiation. Diversity is most naturally thought of as a measure of how heterogeneous a system is-in the case of genetic diversity, for example, it might be a measure of how many different alleles there are in a population, either at some particular locus or on average across all (or some set of) loci. Differentiation, though, is a measure of how different two things are. Two populations that share most of their common alleles are relatively undifferentiated, whereas two that do not share many alleles will be quite highly differentiated. Note that given this distinction, diversity and differentiation are (broadly) independent of one another. In order to see this, consider the fact that two populations that are both very diverse at some locus (each population has a large number of alleles at the locus or loci in question) can vary from entirely differentiated (sharing no alleles in common) to undifferentiated (each population has the same alleles at the same frequencies at each locus of interest). Moreover, two populations each of which has very low diversity (say, each is fixed for a single allele at the locus of interest) can vary from entirely differentiated (fixed for different alleles) to completely undifferentiated

\footnotetext{
${ }^{4}$ Even in the professional literature, there is substantial confusion surrounding these concepts and associated measures. Consider the following, from a "Correction" published by Kronholm et al. (2010b): "In the introduction of Kronholm et al. (2010a), we discuss what properties a differentiation measure, like $\mathrm{F}_{\mathrm{ST}}$, should or was assumed to have. Recent developments have shown that $F_{S T}$ in fact does not have these properties." The authors cite and thank Jost, who we also believe has helped identify and publicize some of the conceptual difficulties and problematic assumptions associated with these measures.

${ }^{5}$ Ecological genetics has been more explicit about these distinctions (n.b., Lou Jost is an ecological geneticist), perhaps because it has to be clear about whether token numbers or token frequencies, within or across types, for genes or for species, are being measured. For example, a textbook in the field dedicates a whole chapter to "Genetic diversity and differentiation" (Lowe et al. 2004, Ch. 3). Some of the distinctions drawn are broadly similar to those we prefer, e.g., they note that "Genetic diversity measures estimate the amount of variation that is found in a population, while genetic differentiation measures describe how this variation is partitioned among populations." However, we suggest a narrower definition of differentiation than the one Lowe et al. actually employ, and believe it is important to more carefully distinguish measures of heterozygosity from other measures of variation.
} 
(fixed for the same alleles). And, while it is obvious that two populations which differ with respect to their diversity (where one, say, is very diverse at the relevant loci and the other is fixed for a single allele at all the relevant loci), cannot be entirely undifferentiated, their degree of differentiation can still vary from relatively low to complete (consider the case where one population is fixed for a particular allele at a locus, and the other population has ten alleles at that locus, but where the most common one is that on which the first population is fixed).

Measures of diversity and differentiation can be usefully contrasted with measures of heterozygosity. Heterozygosity measures (which include Wright's $F_{\mathrm{ST}}$, Nei's closely related $G_{\mathrm{ST}}$, and the Shannon Entropy measure) look to the fraction of heterozygotes (as opposed to homozygotes) in a population. These measures can be used to compare expected heterozygosity (via allele frequencies and assumptions about Hardy-Weinberg equilibrium) to observed heterozygosity in order to tease out aspects of population structure. But heterozygosity measures reflect both population diversity and the differentiation among populations. As such, they often cannot distinguish differences that are the result of different degrees of differentiation from differences that are the result of different degrees of diversity.

As a short case study in how and why these aspects of genetic variation are not the same, consider again Lewontin's (1972) results. His result (that $85.4 \%$ of genetic variation is found within populations, and only $6.3 \%$ is found between races) was generated by estimating within and between population "diversity" using the Shannon Entropy index (see below). As Lewontin himself noted, this measure is closely related to Wright's $F$-statistic, $F_{\mathrm{ST}}$ and its multi-locus extension, $G_{\mathrm{ST}}$, and behaves similarly. Lewontin refers to all of these as measures of "diversity," and considers them to be estimates of the "allocation of... human genetic diversity" within and between populations (p. 396). But, based on the above taxonomy, measures of heterozygosity (including Shannon Entropy, $F_{\mathrm{ST}}$, and $G_{\mathrm{ST}}$ ) are not, strictly speaking, measures of diversity at all. ${ }^{6}$

Why does it matter that the measures Lewontin employed are not strictly speaking measures of diversity, but rather measures of heterozygosity? Consider for example one of the conclusions that Lewontin draws from his analysis, namely that "[if] everyone on earth became extinct except for the Kikiyu of East Africa, about $85 \%$ of all human variation would still be present in the reconstituted species." (Lewontin et al. 1984, p. 126). This claim is often repeated in the literature (see, e.g., Wetherell 1996, pp. 183-184; Giuliano et al. 2007, pp. 6-7), and is generally viewed as following directly from the claim that (on average) $85 \%$ (at least) of human genetic "diversity" is

$\overline{6}$ This point is made forcefully by Jost (2008). within-population "diversity." The problem is that a measure of population heterozygosity, and the associated heterozygosity partitions, do not license claims about population diversity, and hence facts about the heterozygosity partition cannot support any reasonably intuitive understanding of what it would mean to say that "about $85 \%$ of all human variation would still be present in the reconstituted species."

To see this, imagine two populations, each of which has 10 equally frequent alleles at a locus (so both populations are very diverse) but where the populations share no alleles in common (the populations are completely differentiated). In this case, interpreted as "diversity," the Shannon Entropy measure returns the result that about $77 \%$ of the "diversity" exists within each population, and only $23 \%$ is "between" populations; $G_{\mathrm{ST}}$ gives a more extreme but qualitatively similar result (94\% and $6 \%$ ). But the two populations share no alleles in common; as each has 10 alleles, eliminating one of the two populations would eliminate a full half the diversity (on a plausibly intuitive understanding of diversity, anyway), not $23 \%$ (nor $6 \%$ ). Note that the fact that the two measures show low "between" population measures is a consequence of the fact that the measures are being used to partition the total genetic variation (in this case, the heterozygosity) into the portion of the variation within and portion of the variation across populations. A partition, in this sense, is a fragmentation of the total genetic variation and hence high within population variation necessitates that between population variation will be low. ${ }^{7}$

We turn now to unpacking, at least partially, the nature of genetic diversity measures, genetic differentiation measures, and heterozygosity measures, as well as the relationship between these. This is a first step in our efforts to encourage a more careful approach to the importantly different senses of "genetic variation." We explore the (i) definition, (ii) key features, (iii) sample equations, and (iv) uses of, respectively, (1) genetic diversity, (2) differentiation, and (3) heterozygosity.

\footnotetext{
7 This kind of example is used by Jost (2008) and others to show why measures like $G_{S T}$ should not be interpreted as measures of diversity. The equations for calculating the Shannon Entropy measure and $G_{S T}$ are below, and it is a worthwhile exercise to compute the heterozygosity partitions for a variety of circumstances in order to get a sense of how the measures in fact behave. Note that for loci with relatively low overall diversity - which were the kind that Lewontin's 1972 analysis in fact focused on-measures like the Shannon Entropy measure and $G_{S T}$ do not usually produce such misleading results, and so this should not be taken to imply that the estimates of within- and between-population diversity (in the sense we describe below) in humans are necessarily very different than that implied by Lewontin's analysis. Nevertheless, the analysis used is inappropriate if one is trying to measure diversity.
} 
Genetic Diversity and Diversity Partitions: Sense 1 of "Genetic Variation"

\section{Definition}

Genetic diversity is the amount of genetic difference or variation in a population. Genetic diversity measures can be relatively more or less sensitive to the frequency of the alleles in question. At the limit, a genetic diversity measure may simply count the number of alleles, and ignore the frequency of them altogether.

A diversity partition compares the genetic diversity in sub-populations to the genetic diversity in the population as a whole; again, depending on the measures chosen, these comparisons will take account of allele frequencies to different degrees.

\section{Key Features}

Diversity: Ceteris paribus, a population is more (rather than less) diverse if:

- it has more alleles per locus, and;

- the frequency of alternative alleles are more rather than less equal (but note that this is suppressed when a simple allele count is used)

Diversity Partition: For a diversity partition, the "raw count" diversity of a sub-population (total number of alleles) can only be equal to or less than that of the population as a whole; however, when gene frequencies are taken into account, no particular relationship must hold between the diversity of the parts and the diversity of the whole (the diversity of the whole can be less than, equal to, or greater than the diversity of the parts).

\section{Sample Equations}

There are a number of measures that respect the definition and key features of diversity measures and the associated diversity partitions. Here, we take as an example the formal measure championed by Jost, known as Jost's $D .{ }^{8}$

Genetic Diversity: Jost (2008) provides the following "general" diversity measure (Jost's $D$ ):

${ }^{q} D=\sum_{i=1}^{k}\left(p_{i}^{q}\right)^{\frac{1}{1-q}}$

\footnotetext{
${ }^{8}$ While Jost's $D$ has several features that make it a convenient example of a diversity measure, our use of it as an example should not be taken as an endorsement of it as the only or the best measure and associated partition. For discussion, see e.g., Hoffmann and Hofmann 2008, Gabriele et al. 2010, Whitlock 2011, and cites therein.
}

where there are $k$ alleles at a locus, and $p_{i}$ is the frequency of the $i$ th allele, and $q$ controls the sensitivity of the measure to the allele frequency.

So, when $q=0$, Jost's "Zeroth order diversity," ${ }^{0} D$, is

${ }^{0} D=\sum_{i=1}^{k}\left(\left(p_{i}\right)^{0}\right)^{0}$

or simply $k$, or the number of alleles. This is the "raw allele count" interpretation of genetic diversity.

The limit for $D$ as $q$ approaches 1 gives a measure that is somewhat sensitive to allele frequencies (it also has the form of the exponential of Shannon Entropy).

${ }^{1} D=\exp \left(\sum_{i=1}^{k}\left(\left(p_{i}\right)\left(\ln p_{i}\right)\right)\right)$

And, when $q=2$,

${ }^{2} D=\sum_{i=1}^{k}\left(\left(p_{i}\right)^{2}\right)^{-1}$

which heavily discounts rare alleles and gives what is sometimes called the "effective number of alleles."

Consider a case where two populations have an unequal numbers of alleles at a given locus. There is no straightforward answer to a question like "does a locus with 3 alleles of equal frequency have more or less diversity than a locus with one very common allele and a dozen very low-frequency alleles?" Indeed, the diversity measure can reverse, depending on how much one discounts rare alleles as compared to common alleles. This is a clear example of how metrics can be massaged to give a desired result. Alternatively, to make a different and less blunt point, this case clarifies how data interpretation depends critically on metric chosen.

Diversity Partition: Again, a diversity partition is a way of partitioning the extant diversity in a population; it is a measure of how much of the extant diversity occurs, for example, within subpopulations and how much occurs between subpopulations. For the above interpretations of genetic diversity, within- and between-population diversity can be computed as follows (Jost 2008):

${ }^{\mathrm{q}} D_{\mathrm{T}}={ }^{\mathrm{q}} D_{\mathrm{S}} *{ }^{q} D_{\mathrm{ST}}$

where ${ }^{\mathrm{q}} D_{\mathrm{T}}$ is the total diversity; ${ }^{\mathrm{q}} D_{\mathrm{S}}$ is within subpopulation (average); ${ }^{\mathrm{q}} D_{\mathrm{ST}}$ is between subpopulations.

\section{Uses}

Diversity measures are most obviously useful in conservation biology. Which are the genetically most diverse species (or subpopulations) on which we should focus our limited conservation effort resources? One difficulty is that 
depending on how sensitive to allele frequencies the diversity measure deployed is, different sub-populations of the population as a whole may count as the "most diverse," and the amount of "diversity" lost with the loss of a particular sub-population will similarly vary. However, any measure of diversity that reflects the above definition and key features will behave in more predictable ways than will the heterozygosity measures discussed below. In the example above, where two populations each have 10 alleles at equal frequency at a loci, all of which are unique to that population, the expected result, that the entire population has twice the diversity of either of the sub-populations, is achieved (since the allele frequencies are the same, the sensitivity of the measures to allele frequencies makes no difference at all in this, just as one would expect).

Genetic Differentiation: Sense 2 of "Genetic Variation"

\section{Definition}

Genetic differentiation is a measure of how different two populations are, genetically. Genetic differentiation measures, like genetic diversity measures, vary in how sensitive they are to allele frequencies. At one limit, a measure of genetic differentiation may simply count the number of unique alleles in each population (perhaps compared to the number of alleles that are shared). Or the measures might take account of different allele frequencies of shared alleles, counting those populations whose frequencies of shared alleles vary more as more differentiated than those with more similar frequencies.

\section{Key Features}

Ceteris paribus, two populations are relatively more (rather than less) differentiated if:

- the two populations share relatively few alleles in common (relatively many unique alleles rather than relatively many shared alleles)

- the frequency of those alleles that are shared between the two populations are relatively more different (rather than being relatively more similar). Again, this latter condition will be suppressed by measures that discount sensitivity to allele frequency.

\section{Sample Equations}

At the limit, a differentiation measure that is insensitive to differences in (non-zero) frequencies might simply count the number of unique alleles in each population, count the alleles shared by the two populations, and take the differentiation to be the ratio of the (average) number of unique alleles to the total number of alleles.

In order to account for differentiation in the frequencies of common alleles, one might for example consider the difference in the frequency of each allele $p$ at a locus $i$ in two populations (keeping in mind that the frequency of an allele in either population might be zero). So, for two populations with $k$ total alleles (the sum of the number of distinct alleles at that locus in two populations), where $p 1_{\mathrm{i}}$ is the frequency of the $i$ th allele in population 1 and $p 2_{i}$ is the frequency of the $i$ th allele in population 2 :

Differentiation $_{1}=\sum_{i=1}^{k}\left|\frac{\left(p 1_{i}-p 2_{i}\right)}{2}\right|$

This measure can be interpreted, given certain assumptions (see below), as a measure of genetic distance (in this case, Prevosti Distance; see McDonald 2008).

The following equation discounts the effect of rare alleles; given certain assumptions, it can be interpreted as another measure of genetic distance (Euclidean Distance, McDonald 2008):

Differentiation $_{2}=\sqrt{\left(\sum_{i=1}^{k}\left(p 1_{i}-p 2_{i}\right)^{2}\right)}$

Again, it is worth stressing that one cannot say which set of a pair of populations are more differentiated without deciding on a measure. Two populations that share highfrequency alleles but differ dramatically in their lowfrequency alleles (have many unique low-frequencies alleles per locus) will on some measures count as more and on other measures as less differentiated than two populations that share a small number of alleles per locus, but where the frequency of those relatively common alleles varies significantly between the populations.

Uses

Interestingly, measures of differentiation are rarely used directly. While measures of differentiation are conceptually very closely related to diversity partitions, diversity partitions are generally preferred when researchers wish to explore between-population genetic variation. Like Joststyle diversity partitions, for example, differentiation responds linearly with respect to increases in within-population diversity (this is important, because heterozygosity measures do not respond in such a predictable way). Unlike Jost-style diversity partitions, differentiation measures are not relative to the diversity in the two populations. That is, the results are absolute, and not comparative. And this also explains why there can be no "differentiation partition." Differentiation, being a measure of how different two 
populations are, does not lend itself to being partitioned into between- and within-population components. Rather, differentiation, when used with assumptions that permit it to be interpreted as a measure of genetic distance, encourages the recursive nesting of populations. This is what makes it so useful for producing tree-structures and phylogenies. It is clear why, despite having close ties to diversity measures, disparity measures are conceptually distinct. It is also clear why they are rarely used directly-there are relatively few purposes for which an absolute (rather than relative) measure of differentiation is biologically informative.

Genetic Heterozygosity and Heterozygosity Partitions: Sense 3 of "Genetic Variation"

\section{Definition}

Heterozygosity measures are based on the likelihood of a member of a population having different alleles at a given locus. Alternatively it is the likelihood that two members of a population will have different alleles at a given locus.

Heterozygosity Partitions: The observed heterozygosity in a population is often compared to the expected heterozygosity, where the latter assumes Hardy-Weinberg equilibrium. Indeed, the measured heterozygosity of the subpopulation in question compared to the measured heterozygosity of the population as a whole is the heterozygosity partition.

\section{Key Features}

\section{Heterozygosity Measures:}

Ceteris paribus, measures of heterozygosity in a population should increase with:

- the number of alleles per locus

- the evenness of the distribution of the alleles (relatively equal frequencies of alternate alleles increase heterozygosity compared to relatively unequal frequencies of alleles)

In both these cases, the increase in expected heterozygosity is captured by Hardy-Weinberg equilibrium.

Measures of heterozygosity in a population should, ceteris paribus, decrease with:

- the degree of inbreeding, or, more generally, with increased internal population subdivision of any sort (i.e., the Wahlund effect)

Finally, selection can either increase or decrease heterozygosity. In general, directional selection on an allele will result in increased homozygosity, selection for rare alleles will increase heterozygosity, and selection on alleles where there is heterozygotic advantage can (but need not) increase heterozygosity. Drift tends to reduce the number of unique alleles, and hence decreases heterozygosity. Mutation tends to increase the number of unique alleles, and hence tends to increase heterozygosity.

Heterozygosity Partitions: Methods for partitioning heterozygosity into between- and within-population components exploit deviations from Hardy-Weinberg equilibrium to infer population structure. In these cases, an excess of homozygotes in the population as a whole is taken to imply a structured population. Note that these methods must therefore assume that, e.g., selection on the alleles at the locus in question is absent (of course, neutral loci can be and are deliberately chosen for these purposes).

\section{Sample Equations}

The heterozygosity of a population with $k$ alleles at a locus in Hardy-Weinberg equilibrium can be calculated as

$H=1-\sum_{i=1}^{k}\left(p_{i}\right)^{2}$

where $p_{i}$ is the frequency of the $i$ th allele.

Shannon's Entropy measure is closely related, and returns a measure closely related to the heterozygosity of the population (see Lewontin 1972 and cites therein):

$H_{\text {Shannon }}=-\sum_{i=1}^{k}\left(p_{i} \ln p_{i}\right)$

The average heterozygosity for a population can be computed by calculating $H$ or $H_{\text {Shannon }}$ for each locus, and then finding the average for all loci. Note that these measures are absolute, not relative, measures of heterozygosity at a locus.

The classic heterozgosity partition is Wright's $F_{\mathrm{ST}}$ and the multi-allele extension $G_{\mathrm{ST}}$ attributed to Nei:

$G_{\mathrm{ST}}=\frac{\left(H_{\mathrm{T}}-H_{\mathrm{s}}\right)}{H_{\mathrm{T}}}$

where $H_{\mathrm{T}}$ is the expected heterozygosity of population as a whole, and $H_{\mathrm{S}}$ is the average observed heterozygosity of the sub-populations making up $H_{\mathrm{T}}$.

Notice that if there is no population structure (if the subpopulations are identical in composition), $G_{\mathrm{ST}}=0$ (i.e., no excess of homozygotes). If, on the other hand, the two populations are fixed $\left(p_{1}=1\right)$ for two different alleles, $G_{\mathrm{ST}}=1$ (i.e., excess of homozygotes is at a maximum).

Uses

Wright developed the $F$-statistics and associated measurements as part of his Shifting Balance Theory of speciation (e.g., Wade 1992). These measures, then, were fundamentally tied to his interests in the dispersal, isolation, 
selection, and evolution of sub-populations (see Provine 1986, p. 376ff). A major question that Wright sought to answer was whether, at the level of individual groups (demes), there would be sufficient inter-group variation on which the second group-selection phase of the shifting balance process could act (Provine 1986, p. 377). Because inbreeding increases inter-group variation and diminishes intra-group variation, a measure of the degree of inbreeding (i.e., degree of heterozygosity loss; homozygosity excess) was particularly valuable.

For these reasons, a measure that focused on deviations from total population Hardy-Weinberg equilibrium made obvious sense. What is likely to vary with the kind of dispersal envisioned by Wright are the frequencies of alleles that will mostly be present in both populations. Indeed, Wright found variation in heterozygosity to be critically important because of his ongoing interest in epistasis. The phenotypic (and selective) effect of an allele in a given population will depend on the allele frequencies of relevant background genes. That is, changing allele frequencies at one gene changes the effect size and possibly sign at another (Wade 2002). Under those conditions, a measure of heterozygosity that is most adequate for relatively common alleles shared between the populations will generally be nonproblematic. While $F_{\mathrm{ST}}$ and $G_{\mathrm{ST}}$ are sensitive to both the total number of alleles per locus as well as the frequency of those alleles, given Wright's goals in developing these measures (i.e., assess the potential for group selection), the interest in the measures was really in their sensitivity to the differences in the frequencies of relatively common alleles. This sensitivity is most pronounced when the total number of alleles per locus is relatively small, but of course, the loci used can be chosen to have these properties in order to better explore the kinds of changes in population structure that were of particular interest to Wright.

When applied to low-diversity loci, measures of heterozygosity can provide important insights into population structure. But as Jost forcefully notes, it is a mistake to interpret heterozygosity partitions as measures of differentiation or as diversity partitions (Jost 2008). Since the heterozygosity measures deployed are sensitive to both the within-population diversity and population differentiation, very different population structures can lead to similar heterozygosity partitions. Imagine, for example, the $G_{\mathrm{ST}}$ parallel of the result reported by Lewontin-that is, that about $85 \%$ of the expected heterozygosity occurs within the sub-populations, and only about $15 \%$ of the expected heterozygosity occurs between the sub-populations. The sub-populations in this case might have all their alleles in common (and differ in the frequency of those shared alleles), they might have no alleles in common (they might both have a large number of alleles at each loci, but not share any), or anything in-between. In other words, as noted above, heterozygosity partitions can (and do) vary somewhat independently of diversity partitions. So while heterozygosity partitions are sometimes used in, e.g., conservation biology, it is generally a mistake to do so, since it is impossible, without substantial additional information, to say what a particular loss of heterozygosity would entail for the loss of diversity (see Jost 2008, p. 4019). For similar reasons, it is a mistake to treat heterozygosity partitions as measures of differentiation, since, again, pairs of populations that are very different in terms of their differentiation may have the same within- versus between-heterozygosity partitions.

These three measures and senses of genetic variationdiversity, differentiation, and heterozygosity — can be used to describe and explore various aspects of genetic variation in populations. Again, the projects vary, and include describing the relatedness of populations (phylogenetics), exploring internal population structure, assessing the diversity of a particular population or sub-population, either for the purposes of conservation or in order to assess the evolvability of the population, characterizing the differences between populations that are neutral, and distinguishing those from differences that are of adaptive significance, among others.

Genetic distance, for example, is used to characterize distance between populations. Generally, each population is assigned to a point in multidimensional genotypic space, i.e., in terms of genotypic means. Then the genetic distance between pairs of populations is assessed (sometimes literally in Euclidean distance, as in Cavalli-Sforza and Edwards 1967, or at other times as a simple measure of the difference in gene frequencies, transformed in some way, as in Nei; see McDonald 2008). Here, the basic idea is that the distance is an indication of the historical relatedness of the populations: generically close populations will tend to be historically closely related (and this can be represented in a tree diagram, once genetic distances have been established). Genetic distance measures, as a general class of measures, are not necessarily the same as measures of differentiation, nor are they identical to (any particular) heterozygosity partitions nor to diversity partitions. Different measures of genetic distance rely on different aspects of genetic variation. In general, which aspects of genetic variation a particular measure of genetic distance uses will depend on the assumptions made regarding the mechanisms by which the populations diverged. ${ }^{9}$ And one cannot read historical relationships directly off of any measures of genetic variation, because measures of genetic

\footnotetext{
${ }^{9}$ Nei's standard distance, for example, assumes that populations diverge because of genetic mutations and drift, whereas Reynold's distance assumes only drift and purposefully excludes mutation. See Libiger et al. (2009).
} 
variation do not, and cannot, take into account the problem of homoplasy (convergence) nor the closely related phenomena of "back-mutations" and saturation.

While the focus in the technical literature on "the biology of race" has been on population genetics, and on the measures of genetic variation within and between populations, any thorough analysis of the matters at stake would require an investigation of, at the very least, systematics and ecology. The methods used to uncover and understand migration patterns, to make inferences regarding phylogenetics, and to perform clustering analyses, require both the formal methods to unpack the various kinds of genetic variation outlined above, and, importantly, additional information from a variety of other biological, anthropological, archaeological, and linguistic fields. Indeed, a full analysis of the modeling strategies and theoretical context involved would require exploring not only the metrics above, but tracking the role of ontological assumptions, and formal methodology for a variety of cases. ${ }^{10}$ Now, the arguments surrounding the relationship between "race," as a social category, and human population structures, have tended to rely on one, or a very few, measures of genetic variation. The limited nature of these measures is one reason to be suspicious of any attempt to ground a strong ontological category like "race" on these approaches. That social discourse may attempt to justify its claims in the biological theory, but doing so is unjustified and amounts to a pernicious reification. It is to that discourse that we now turn.

\section{Ontological Excesses and Biological Theory}

Different measures of human genetic variation can be legitimately used for different purposes; a measure employed to understand one aspect of human genetic variation (say, a heterozygosity partition) might be inappropriate if employed in an attempt to study another feature (say, the distribution of genetic diversity). Given that the very measures employed, and the range of their legitimate purely technical uses, remains contentious, it is hardly surprising that attempts to draw conclusions about the biological reality of complex and morally laden social categories like "race" from these measures are unstable and contested. In what follows, we suggest that equally valid theoretical interpretations of biological data can be used to justify the existence or non-existence of human races. In other words, the biological data cited in support of claims regarding "race" in fact fail to distinguish between competing claims. The

\footnotetext{
10 The SMEO-P model-i.e., "set-up, mathematically manipulate, explain, objectify-pluralize"-developed in Winther (2006) is useful here.
}

theory, data, and ontology of biology underdetermines social classification and social ontology. ${ }^{11}$

Consider the two episodes we started with: Livingstone versus Dobzhansky circa 1962 and Edwards versus Lewontin circa 2003. Livingstone begins by citing biological data about the distribution of human genetic variation, and moves to a conclusion about the nonexistence of race as an explanatory biological category. He suggests that any attempt to redefine "race" to accord with the biological facts would fail to respect both the ordinary biological and ordinary social uses of the term. Dobzhansky too cites biological data; he argues that while any difference in allele frequencies between populations is sufficient to call those populations "races," that races are only worth naming if those allele frequency differences align across multiple loci. But since they assuredly do in the human case, Dobzhansky argues, it makes no sense to deny either the existence, nor the taxonomic significance, of human races.

Again, concerning assumptions about the nature of patterns of human genetic variation, Livingstone looks for sharp discontinuities, and, failing to find any, declares the human species to be "race free." Dobzhansky looks to clustering, and finding clusters (or at least having good reasons to think that clusters must exist), declares race to be biologically real in the human case.

Fast forward. Lewontin (1972) is widely interpreted as demonstrating that "race" was not a biologically real category. Lewontin perhaps encouraged, and certainly did nothing to discourage, this interpretation of his results. Again, Lewontin's argument starts with biological factsin this case, that given one way of partitioning genetic variation, most (over $85 \%$ ) of the genetic variation (according to the Shannon Entropy measure) was withinpopulation variation, and only a small fraction (a bit over $6 \%$ ) was between races. He concludes that "race," as either an explanatory or classificatory biological category, is "virtually" worthless. Edwards, in 2003, looks at (roughly) the same data, but argues that if one looks for clusters (rather than merely partitioning the variation), one will find races staring back. Races, Edwards argues, are biologically real, and indeed, are worthy of biological classification; Edwards remains agnostic with respect to the extent that the biologically real races uncovered will also

\footnotetext{
11 These debates surrounding the ontology of socially identified races take place against a background where basic life prospects (income, life expectancy, morbidity, standards of living, and quality of life more generally) are strongly correlated with socially identified race. The question: "What accounts for these differences?" lurks behind these debates, but the implications of that question are not always obvious, nor is it obvious what the answers to that question the various positions in the debate suggest. But at least some of the dangers in biological explorations of human population structure that, e.g., Kitcher (2007) points towards, and that Kaplan (2010) highlights, are wrapped up in these issues.
} 
be biologically explanatory, but does not dismiss the possibility out of hand. Again, if even the biological ontological conclusion is underdetermined by biological theory and data; the social ontological conclusion will certainly be underdetermined too, given that the complexity of social agendas and assumptions will also influence the latter sorts of conclusion.

\section{Conclusion: Towards an Objectivist Constructivism}

So, can the biological data support any particular racial ontology? We argue that it cannot. At one extreme, if there was no population structure at all in humans, if all loci varied strictly independently from one another, it would be difficult to defend a racial realist position ${ }^{12}$ as Mills defines it (see above and Fig. 1). But no one has ever seriously suggested that there is no population structure at all in humans, or that all (genetic) traits vary strictly independently from each other across our species. And if there is any structure at all, there is room for a racialist to argue that it is that structure that makes races "real." Lewontin's result that only about $6 \%$ (whatever this number means, see above) of the variation in the species occurs between the races is insufficient to make racialism impossible; $6 \%$, after all, is not zero.

But the biology usually cited is of no use to the realist, either. The ability to use clustering software to recapitulate racial categories based on ancestral continent of origin, or to find tree-like relationships among populations, does not make the clusters found biologically real races, or the branches of the trees found clades (nor, pace Andreasen $(2000,2004)$ would a population's being a clade make that population into a race). At the very least, the full-blown racial realist ought to be able to provide a consistent and clear biological justification for treating some clusters (or branching points on a tree) and not others as races and assigning to those clusters (but not others) a particular kind of biological meaning. After all, the same formal techniques-indeed, the same software packages—can (and do) identify populations that are not generally considered races within the context of contemporary U.S. discourse ("the Dutch" for example; see Novembre et al. 2008; see Kaplan 2010 for discussion; Kitcher 2007 makes a similar point, pp. 304-306). Certainly nothing in the techniques that use genetic variation to explore population structure can provide that justification, and it is vanishingly unlikely that anything in biology can.

Recall our summary of the possible ontological positions one can take about race, as outlined by Mills (see

\footnotetext{
12 We ignore here the complexities of developmental biology and non-genetic heritable resources.
}

Fig. 1). Of these positions, the objectivist constructivist position strikes us as by far the most plausible. ${ }^{13}$ And here, biological facts-at least those that emerge from population genetics and the study of genetic variation-are simply irrelevant. Certainly biology isn't irrelevant-for example, the mechanisms by which the health disparities (a biological fact if ever there was one! see Kaplan 2010 for discussion) between black and white Americans are produced are clearly relevant to understanding the roles that race plays and the kind of thing that race is. But that is a far cry from being able to use biological facts about populations as part of our definition of race, or as part of our racial classifications. The hope that one of a variety of measures that emerge from particular areas of biology would provide the theoretical foundations for either the rejection of races or for the affirmation of the reality of races fails.

Whence the failure? It is not because the measures are "wrong" or even useless. Rather, there are many metrics and meanings of "genetic variation." Our choice among these is conventional, and the sort of racial landscape provided by one metric may be shifted, even reversed, by another. Do we choose to focus on the ways in which even relatively small differences can be leveraged to find clusters that reflect population structure, and argue from there that the clusters we find are races? Or do we note the hierarchical nature of the clusters generated using these methods, and declare that these methods cannot find races unless we already know what kind of cluster (what size of cluster, and how many clusters) we are looking for? Do we focus instead on the clinal nature of gene-frequency variation at most loci, and argue that clusters only look like they have sharp boundaries and hence look like races because our sampling encourages that view? Or, again, do we choose to focus on the relative paucity of genetic variation in our species, and hence the relative genetic similarity of all humans, and argue that the population structure that exists is not based on sufficient genetic differences to warrant calling these "populations" in any interesting sense? None of the moves from the measure to the claim about "race" is justified; however, all the biological measures are legitimate, and all exploit the same basic biological facts.

The technical tools provided by biological theory are diverse, but the social house we build is of our own choosing. It seems time to accept honestly our burden of conceptualizing "race," and not allow ourselves to be imprisoned by formal abstractions. Biological theory and data do not force the "race" concept upon us; we force it upon ourselves, to our own detriment.

\footnotetext{
${ }^{13}$ In various forms, this position, or one very much like it, has been defended by Appiah and Gutmann (1996), Hacking (2005), Haslanger (2010), Omi and Winant (1989), and others.
} 
Acknowledgments Matthew Kopec, Michael J. Wade, and the editors of this special issue of Biological Theory, Kim Sterelny, Massimo Pigliucci, and Werner Callebaut, kindly provided feedback on this paper. Kaplan would like to thank the participants at the KLI "The Meaning of 'Theory' in Biology" workshop for their comments, and the Oregon State University Center for the Humanities, whose support helped make this work possible. Winther thanks Ian Hacking, Carlos López-Beltrán, and Amir Najmi for ongoing discussions on these topics. Kim Sneppen's support of Winther's guest research stay at the Biocomplexity Center, Niels Bohr Institute, University of Copenhagen, is also gratefully acknowledged. This paper, written fully jointly, with authors listed alphabetically, is the result of an ongoing collaboration that emerged from an e-mail exchange between the authors that began with Kaplan reading Winther (2011).

\section{References}

Andreasen RO (2000) Race: biological reality or social construct? Phil Sci 67:S653-S666

Andreasen RO (2004) The cladistic race concept: a defense. Biol Philos 19:425-442

Appiah KA, Gutmann A (1996) Color conscious: the political morality of race. Princeton University Press. Princeton, NJ

Cavalli-Sforza LL, Edwards AWF (1967) Phylogenetic analysis: models and estimation procedures. Am J Hum Genet 19:233-257

Cognato AI (2007) A standard DNA taxonomy for insects? USDA Forest Service Proceedings RMRS-P-45, pp 11-12

Edwards AWF (2003) Human genetic diversity: Lewontin's fallacy. BioEssays 25:798-801

Giuliano P, Spilimbergo A,Tonon G (2007) Genetic, cultural and geographical distances. IZA Discussion Paper No. 2229. http:// www.economics.harvard.edu/files/faculty/97_Genetics_August 2007.pdf. Accessed 3 Nov 2011

Hacking I (2005) Why race still matters. Dædalus 134(1):102-116

Haslanger S (2010) Language, politics, and "the folk": looking for "the meaning" of "race". Monist 93:169-187

Hoffmann S, Hoffmann A (2008) Is there a "true" diversity? Ecol Econ 65:213-215

Jost $\mathrm{L}$ (2008) $\mathrm{G}_{\mathrm{ST}}$ and its relatives do not measure differentiation. Mol Ecol 17:4015-4026

Kalinowski ST (2011) The computer program STRUCTURE does not reliably identify the main genetic clusters within species: simulations and implications for human population structure. Heredity 106:625-632

Kaplan JM (2010) When socially determined categories make biological realities: understanding Black/White health disparities in the U.S. Monist 93:281-297

Kaplan JM (2011) "Race": what biology can tell us about a social construct. In: Encyclopedia of life sciences. Wiley, Chichester. doi:10.1002/9780470015902.a0005857

Kitcher P (2007) Does "race" have a future? Philos Public Aff 35:293-317

Kronholm I, Loudet O, de Meaux J (2010a) Influence of mutation rate on estimators of genetic differentiation-lessons from Arabidopsis thaliana. BMC Genet 11:33
Kronholm I, Loudet O, de Meaux J (2010b) Correction: influence of mutation rate on estimators of genetic differentiation: lessons from Arabidopsis thaliana. BMC Genet 11:88

Levins R, Lewontin RC (1980) Dialectics and reductionism in ecology. Synthese 43:47-78

Lewontin RC (1972) The apportionment of human diversity. Evol Biol 6:381-398

Lewontin RC, Rose S, Kamin LJ (1984) Not in our genes: biology, ideology, and human nature. Pantheon Books. New York

Li W-H, Sadler LA (1991) Low nucleotide diversity in man. Genetics 129:513-523

Libiger O, Nievergelt CM, Schork NJ (2009) Comparison of genetic distance measures using human SNP genotype data. Hum Biol 81:389-406

Livingstone FB, Dobzhansky T (1962) On the non-existence of human races. Curr Anthropol 3:279-281

Lowe A, Harris S, Ashton P (2004) Ecological genetics. Design, analysis, and application. Blackwell, Oxford

McDonald D (2008) Distances summary. http://www.uwyo.edu/ dbmcd/molmark/GenDistEqns.pdf. Accessed 3 November 2011

Mills CW (1988) "But What Are You Really? The Metaphysics of Race.” Blackness visible: essays on philosophy and race. Cornell University Press, Ithaca, NY, pp 41-66

Novembre J, Johnson T, Bryc K, Kutalik Z, Boyko AR, Auton A, Indap A, King KS, Bergmann S, Nelson MR, Stephens M, Bustamante CD (2008) Genes mirror geography within Europe. Nature 456:98-101

Omi M, Winant H (1989) Racial formation in the United States: from the 1960 s to the 1980s. Routledge, New York

Pritchard JK, Stephens M, Donnelly P (2000) Inference of population structure using multilocus genotype data. Genetics 155:945-959

Provine WB (1986) Sewall Wright and evolutionary biology. University of Chicago Press, Chicago

Rosenberg NA, Pritchard JK, Weber JL, Cann HM, Kidd KK, Zhivotovsky LA, Feldman MW (2002) Genetic structure of human populations. Science 298:2381-2385

Wade MJ (1992) Sewall Wright: gene interaction and the shifting balance theory. In: Antonovics J, Futuyma D (eds) Oxford Surveys of evolutionary biology, vol VI. Oxford University Press, Oxford, pp 35-62

Wade MJ (2002) A gene's eye view of epistasis, selection, and speciation. J Evol Biol 15:337-346

Wetherell M (1996) Identities, groups, and social issues. Sage, London

Whitlock MC (2011) $\mathrm{G}_{\mathrm{ST}}$ and D do not replace $\mathrm{F}_{\mathrm{ST}}$. Mol Ecol 20:1083-1091

Winther RG (2006) Fisherian and Wrightian perspectives in evolutionary genetics and model-mediated imposition of theoretical assumptions. J Theor Biol 240:218-232

Winther RG (2011) ¿La cosificación genética de la "raza"? Un análisis crítico. In: López-Beltrán C (ed) Genes \& mestizos: genómica y raza en la biomedicina Mexicana. UNAM, Mexico City, pp 237-258

Yu N, Chen F-C, Ota S, Jorde LB, Pamilo P, Patthy L, Ramsay M, Jenkins T, Shyue S-K, Li W-H (2002) Larger genetic differences within Africans than between Africans and Eurasians. Genetics 161:269-274 